\title{
Multistrategic Memory Training with the Metamemory Concept in Healthy Older Adults
}

\author{
Jung-Hae Youn ${ }^{1}$, Jun-Young Lee ${ }^{2}$, Seolmin $\mathrm{Kim}^{3}$ and Seung-Ho Ryu ${ }^{3} \bowtie$ \\ ${ }^{1}$ Yongmoon Graduate School of Counseling Psychology, Seoul, Korea \\ ${ }^{2}$ Department of Psychiatry, Seoul National University Boramae Hospital, Seoul, Korea \\ ${ }^{3}$ Department of Psychiatry, School of Medicine, Konkuk University, Konkuk University Medical Center, Seoul, Korea
}

\begin{abstract}
Objective According to the increase of older people, the need for effective methods to maintain or improve cognitive functions in the elderly has increased. These cognitive enhancing methods may contribute to the prevention of elderly cognitive decline by aging and dementing illness as well. This study aimed to examine the effectiveness of multistrategic memory training with the metamemory concept on cognitive functions in the normal health elderly in Korea.

Methods The program used in this study was developed by psychiatrists and psychologists in accordance with Korean situations. We applied the training program to the community-dwelling elderly with subjective memory complaints. Twenty participants were randomly received the intervention with 20 non-treatment controls. This program consisted of 10 sessions and was administered once a week. We examined the effects of this memory training for verbal memory, visuospatial memory, working memory, and verbal fluency ability by repeated ANOVA.

Results There were significant improvements in Word List Short-term Delayed Free and Cued Recall, Word list Long-term Delayed Free and Cued Recall and visuospatial recognition memory. Performance improvements in visuospatial span forwards and the Categorical Fluency Test were also significant. These improvements were still significant after adjusting for depression improvement exact categorical fluency.
\end{abstract}

Conclusion This study shows that multistrategic memory training with the metamemory concept may improve memory ability and other cognitive functions which are not trained and that these improvements may be achieved by pure cognitive training effects.

Psychiatry Investig 2011;8:354-361

Key Words Memory training, Subjective memory complaints, Metamemory, Cognitive aging.

\section{INTRODUCTION}

Memory decline is one of the most common complaints in the elderly. The frequency of subjective memory complaints (SMC) is over $50 \%$ in the Korean elderly population and causes functional impairment in daily life and a poor quality of life in later life. ${ }^{1}$ Therefore, reduction of SMC by a cognitive training program may be beneficial to the healthy elderly.

Several cognitive training programs have been conducted in healthy older people and these cognitive training programs

Received: March 15, 2011 Revised: May 22, 2011

Accepted: May 29, 2011 Available online: November 3, 2011

$\triangle$ Correspondence: Seung-Ho Ryu, MD, PhD

Department of Psychiatry, School of Medicine, Konkuk University, Konkuk University Medical Center, 4-12 Hwayang-dong, Gwangjin-gu, Seoul 143729 , Korea

Tel: +82-2-2030-7567, Fax: +82-2-2030-7748, E-mail: shryu@kku.ac.kr

(a) This is an Open Access article distributed under the terms of the Creative Commons Attribution Non-Commercial License (http://creativecommons.org/licenses/bync/3.0) which permits unrestricted non-commercial use, distribution, and reproduction in any medium, provided the original work is properly cited. have shown improvements in cognitive performance. ${ }^{2-6}$ However, these improvements have been limited to only the trained cognitive area but not to other cognitive areas ${ }^{4,7}$ because most of these cognitive trainings have simply been focused on a single cognitive domain and a single strategy. ${ }^{2,4}$ Moreover, many previous studies have shown that cognitive training programs improve not only cognitive functions but also depressive symptoms. Therefore, it is difficult to determine whether such improvements are due to pure improvement of cognitive functions or depressive symptoms. ${ }^{8,9}$

In the present memory training program, we used multistrategic memory training, including a metamemory approach to overcome the limitation of previous studies. The term "metamemory" was introduced by Flavell. ${ }^{10}$ This term includes knowledge, perception, and beliefs about their own memory and the memory system. ${ }^{11}$ Metamemory emphasizes on self-reflective cognitive process in learning and people's knowledge about their memory. This kind of concept was 
also used in the term "memory self-efficacy" as a specific dimension of metamemory. ${ }^{12}$ Recent studies indicate that metamemory process, including memory monitoring, judgments of learning and control process, can enhance the effectiveness of learning. ${ }^{13,14}$ Moreover, metamemory is positively associated with everyday memory performance. ${ }^{15}$ These results led us expect that knowledge about memory functioning and memory self-efficacy may permit effects on transfer near tasks and every day activities. We hypothesized that this multistrategic memory training with a metamemory approach may improve cognitive performance of untrained areas as well as memory ability and that these effects will be significant after controlling for depressive symptom improvement.

\section{METHODS}

\section{Participants}

The participants of the study were recruited from memory clinics and community-based center for dementia in Seoul. We included healthy people aged over 55 years with SMC. Two psychiatrists trained in neuropsychiatry and dementia research examined each participant according to the clinical assessment battery protocols of the Korean version of the Consortium to Establish a Registry for Alzheimer's disease (CERAD-K). ${ }^{16,17}$ We also administered CERAD-K neuropsychological assessment, the Korean version of the Geriatric Depression Scale, short form (SGDS-K), ${ }^{18}$ and the Seoul Instrumental Activities of Daily Living. ${ }^{19}$

We excluded participants who had dementia, major psychiatric disorders, or serious medical/neurological disorders that could affect their mental functions. Two psychiatrists screened dementia and other major psychiatric disorders based on DSM-IV criteria. ${ }^{20}$ All participants had adequate vision and hearing, although many wore glasses, and some required hearing aids. The study was viewed and approved by the Institutional Review Board of each institution and written informed consent was obtained from each participant. We randomly allocated 20 participants each into the intervention or control group. The control group had not involved in any specific intervention related to cognitive performances. Of the 20 participants in the intervention group, 16 completed the program. Of the 20 participants in the control group, 4 were lost to follow-up.

\section{Training program}

The program used in this study is based on the metamemory concept and a multistrategic approach for the enhancement of memory. The metamemory concept has three phrases; metaknowledge, metamonitoring, and metajugement. Pour knowledge, the participants can obtain information
Table 1. The contents of each session of the training program

\begin{tabular}{cl}
\hline Session & \multicolumn{1}{c}{ Contents } \\
\hline 1 & Introduction of forgetfulness \\
2 & Memory process 1 \\
3 & Memory process 2 \\
4 & Memory structure 1 \\
5 & Memory structure 2 \\
6 & Memory and attention \\
7 & Memory and brain \\
8 & Memory and environment \\
9 & Memory and perception \\
10 & Memory and forgetting \\
\hline
\end{tabular}

what they believe or think about their personal memory performance and collective cognitive aging, about what works in the process of memorizing. Through monitoring, the elders obtain judgments at the metamemorial level about the knowledge or strategies. We offer the practical opportunity to find a distinction between prospective and retrospective monitoring about memory performance by applying multimemory strategies that clarifies the effect of each strategy. The program consists of a total of 10 sessions every week. Each session is comprised of 3 parts, and it takes 90 minutes to complete the session. The goal of the first part of each session is to strengthen metaknowledge, based on theoretical models of the memory process and cognitive aging. All participants were educated through visual stimuli consisting of drawings, charts and pictures provided by professional illustrators and computer graphic designers. We help the elderly understand and accept the concept of cognitive aging, suggest efficient strategies for dealing with cognitive aging and provide guidance on implementing these strategies into their daily lives. The second and third parts of each session focus on metamonitoring, metajugement, memory training via the practice of external and internal strategies. The second part consists of collective memory training, and the third part includes personal memory training. The materials used in memory training are developed through the collaboration of psychiatrists, psychologists and illustrators. The collective memory training is structured so that stimuli can be given through a computer screen. In the personal training, the problems are given on paper, emphasizing metamonitoring. Each session has a main theme based on the specific strategy designated to the session, and each theme focuses on the memory process (Table 1).

\section{Neuropsychological assessment}

All subjects in the intervention and control groups were evaluated before the memory training (pre-test evaluation) and immediately after the memory training (post-test evalu- 
Table 2. Baseline characteristics: demographic, cognitive and psychosocial characteristics

\begin{tabular}{|c|c|c|c|}
\hline Measures & $\begin{array}{l}\text { Intervention group } \\
\qquad \mathrm{N}=16\end{array}$ & $\begin{array}{c}\text { Control group } \\
\mathrm{N}=16\end{array}$ & $\mathrm{p}$ \\
\hline \multicolumn{4}{|l|}{ Demographic } \\
\hline Gender (male: female) & $6: 10$ & $7: 9$ & 0.719 \\
\hline Age (years) & $68.75(4.60)^{*}$ & $69.00(3.45)$ & 0.863 \\
\hline Education (years) & $10.19(3.19)$ & $9.56(2.45)$ & 0.539 \\
\hline \multicolumn{4}{|l|}{ Cognitive } \\
\hline \multicolumn{4}{|l|}{ Verbal memory } \\
\hline Word list memory ${ }^{\dagger}$ & $24.56(6.36)$ & $27.94(4.12)$ & 0.085 \\
\hline Short-term delayed free recall & $4.31(2.15)$ & $5.63(1.71)$ & 0.066 \\
\hline Short-term delayed cued recall & $5.44(2.07)$ & $6.63(1.54)$ & 0.075 \\
\hline Long-term delayed free recall & $3.94(2.24)$ & $5.69(1.74)$ & 0.019 \\
\hline Long-term delayed cued recall & $4.69(2.30)$ & $6.44(1.50)$ & 0.016 \\
\hline Recognition & $24.94(3.24)$ & $27.19(2.34)$ & 0.032 \\
\hline \multicolumn{4}{|l|}{ Visuospatial memory } \\
\hline SRFT copy & $15.38(0.96)$ & $14.69(1.30)$ & 0.099 \\
\hline SRFT immediate recall & $11.97(3.84)$ & $11.31(2.77)$ & 0.584 \\
\hline SRFT delayed recall & $11.22(4.29)$ & $10.75(2.91)$ & 0.720 \\
\hline SRFT recognition & $15.69(2.65)$ & $17.00(1.41)$ & 0.094 \\
\hline \multicolumn{4}{|l|}{ Attention } \\
\hline DST forward & $7.69(1.82)^{\ddagger}$ & $8.19(1.56)$ & 0.410 \\
\hline DST backward & $4.56(1.97)$ & $4.38(1.20)$ & 0.748 \\
\hline VST forward & $6.44(1.63)$ & $7.06(1.34)$ & 0.246 \\
\hline VST backward & $4.44(2.00)$ & $4.75(1.39)$ & 0.611 \\
\hline \multicolumn{4}{|l|}{ Fluency } \\
\hline Phonemic fluency & $25.00(9.68)$ & $20.69(8.89)$ & 0.199 \\
\hline Categorical fluency & $21.19(4.85)$ & $21.88(4.21)$ & 0.671 \\
\hline \multicolumn{4}{|l|}{ Psychosocial } \\
\hline SMCQ & $9.56(2.80)$ & $6.56(2.68)$ & 0.004 \\
\hline SGDS & $10.69(3.00)$ & $6.44(4.35)$ & 0.003 \\
\hline
\end{tabular}

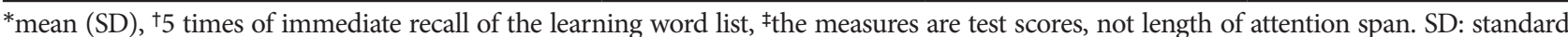
deviation, SRFT: Simple Rey Figure Test, DST: Digit Span Test, VST: Visual Span Test, SMCQ: Subjective Memory Complaints Questionnaire, SGDS: Geriatric Depression Scale, short form

ation). The neuropsychological assessment was conducted by two clinical psychologists who did not participate in the training program. In all evaluations, evaluators were blinded to the cognitive status of each participant and group characteristics. Between the pre-test and post-test evaluation, subjects in the intervention group participated in the memory training program, but those in the control-group did not receive any type of intervention. All participants joined the program free of charge, with no financial reward for taking part in the study. The control group was selected from the waiting list for the training program.

We selected appropriate items from the Elderly Memory Disorder Scale (EMS), which was developed and standard- ized to the Korean population. ${ }^{21}$ To assess memory function, we administered the Elderly Verbal Learning Test (EVLT) and the Simple Rey Figure Test (SRFT), included in EMS. In the EVLT, 9 words from 3 categories are given, and after the patient is administered an immediate recall of the learning word list 5 times, an immediate recall of the interfering word list is administered. In addition, short-term delayed free and cued recall of the learning word lists and long-term $(20 \mathrm{~min})$ delayed cued recall and recognition tasks are included. In the SRFT, copying tasks, immediate recall tasks and tasks on delayed recall and recognition after $20 \mathrm{~min}$ are included. To evaluate attention and executive function, we used the Digit Span Test (DST), the Spatial Span Test (SST), the Phonemic 


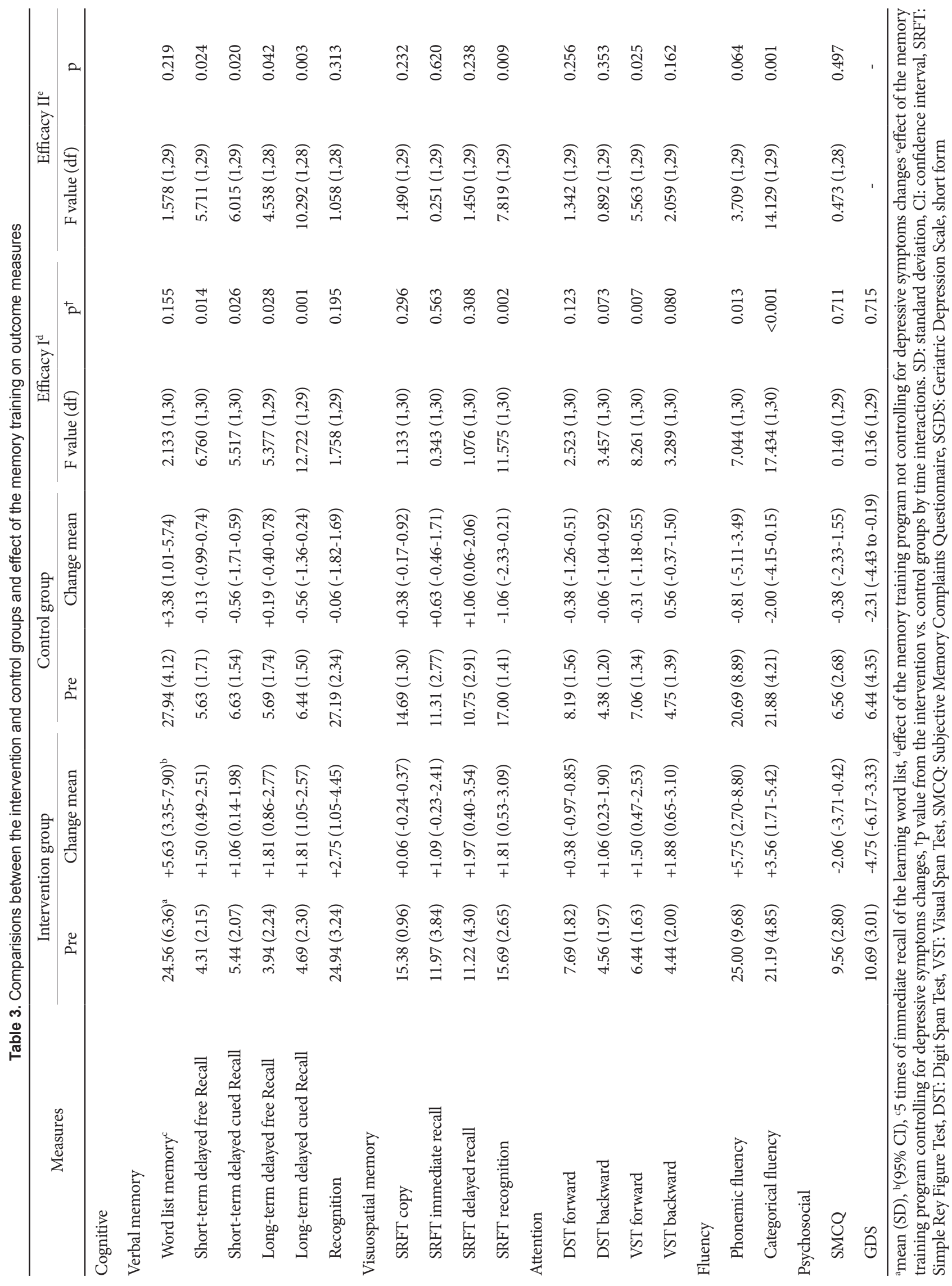




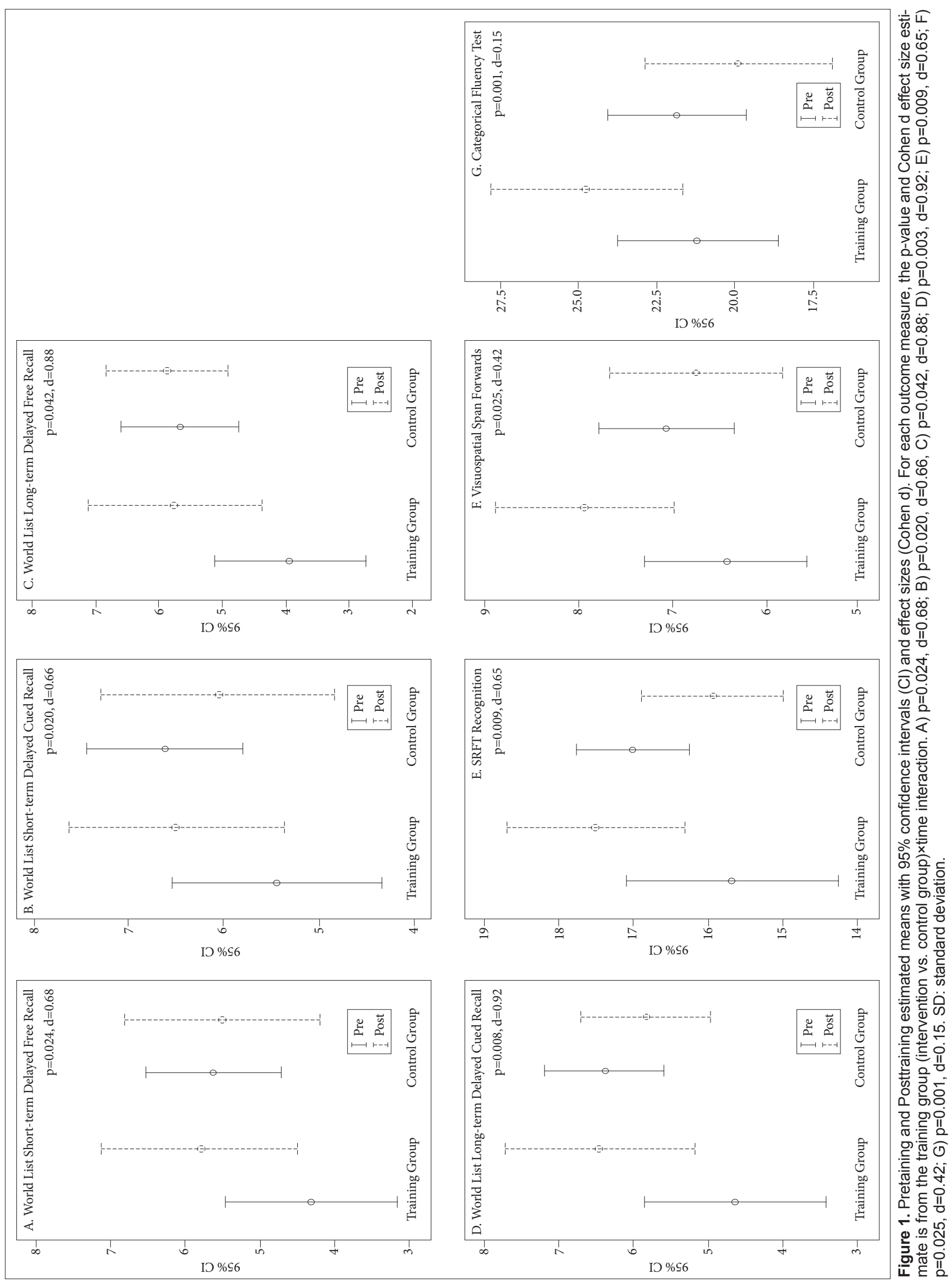


Fluency Test ${ }^{22}$ and the Categorical Fluency Test (CFT) ${ }^{23}$ The DST and SST consist of forward and backward recall. In the categorical fluency test, the patient is asked to name as many animals and fruits as possible for $1 \mathrm{~min}$. In the phonemic fluency test, the patient named as many words as he can that start with 3 Korean letters for $1 \mathrm{~min}$. The Subjective Memory Complaints Questionnaire (SMCQ) was used to evaluate subjective memory functioning. SMCQ includes 15 items and its higher score indicates more perceived cognitive decline. SMCQ is validated for and adapted to the Korean population. ${ }^{24}$ The SGDS-K was used to evaluate depression. ${ }^{18}$

\section{Statistical analysis}

PASW 17.0 for WINDOWS was used for data analyses. A $p$ values of $<0.05$ derived from the two-tailed test were considered statistically significant.

We compared baseline performance between the 2 groups in order to test for possible differences in each of the measures, although individuals were randomly assigned to the groups. The independent samples $t$ test was performed for age, educational level and baseline neuropsychological scores. The Chi-square test was used for analysis of the gender. General Linear Model repeated measures analysis of variance was carried out to examine the effect of the intervention on memory training. Analyses were performed with the group as a between-subjects variable and neuropsychological testing performance on the first and second follow-up phase as the within-subjects factor. In the first set of analyses, several baseline scores, Word List Long Term Delayed Free and Cued Recall, Word List Recognition, SMCQ and SGDS were treated as covariates in this model in order to control for significant baseline group differences. In addition, we set the change in SGDS scores between the 2 phases as a covariate to control the effects of depressive symptoms on cognitive performance.

\section{RESULTS}

\section{Baseline characteristics}

A total of 32 participants were included in the study. There were 16 participants in the intervention and control groups. There were no significant baseline differences in baseline characteristics between the 2 groups except for Word List Long-Term Delayed Free Recall ( $\mathrm{p}=0.019)$ and Cued Recall ( $\mathrm{p}=0.016$ ), Word List Recognition ( $\mathrm{p}=0.032)$, SMCQ $(\mathrm{p}=0.004)$ and SGDS score $(\mathrm{p}=0.003)$. Baseline characteristics are summarized in Table 2.

In baseline analysis, participants in the intervention group were more depressed and concerned about their memory function and also had difficulties with 5 cognitive subtests than those in the control group. Therefore, these 5 baseline scores were treated as covariates in order to examine for significant group differences. Since improvement of depressive symptoms may affect cognitive performance regardless of memory training, the change in the SGDS score was controlled by treating as a covariate.

\section{Effects of memory training on outcome measures}

Changes in scores with time and statistical significance between the intervention and control groups are shown in Table 3. To control for the effects of depressive symptoms on performance and to evaluate training efficacy regardless of it, we set changes in SGDS scores between 2 phases as a covariance. The results indicate that benefits from memory training have been shown in the intervention group. Because this was a preliminary study, an unadjusted $\alpha$-level of $5 \%$ was used. To avoid an unacceptable loss of power, adjustments for multiple comparisons were not made.

There were significant effects favoring the intervention group on verbal memory subtests, including Word List Short term Delayed Free $(\mathrm{p}=0.024)$ and Cued Recall $(\mathrm{p}=0.020)$ as well as Word list Long-term Delayed Free $(\mathrm{p}=0.042)$ and Cued Recall ( $\mathrm{p}=0.003)$. Significant effects on the visuospatial memory subtest and SRFT Recognition ( $\mathrm{p}=0.009$ ) indicate greater improvement in the intervention group. On the attention and verbal fluency, there were significant effects favoring the intervention group on visuospatial span forwards $(\mathrm{p}=0.025)$ and CFT ( $\mathrm{p}=0.001$ ). These findings imply that significant improvements occur after controlling for changes in depressive symptoms.

Figure 1 shows pre-post scores and effect sizes (Cohen d) according to group for Word List Short-term Delayed Free $(\mathrm{d}=0.68)$ and Cued Recall $(\mathrm{d}=0.66)$, Word list Long-term Delayed Free ( $\mathrm{d}=0.88)$ and Cued Recall $(\mathrm{d}=0.92)$, SRFT Recognition ( $\mathrm{d}=0.65)$, Visuospatial span forwards $(\mathrm{d}=0.42)$ and CFT ( $(=0.15)$.

\section{DISCUSSION}

In this study, this multistrategic memory training with a metamemory approach improved both only memory ability and other cognitive abilities and the improvement is significant irrespective of improvement in depressive symptoms. These results indicate that this training may have pure cognitive benefits including transfer effect to different cognitive functions. After training, participants were able to better encode and retrieve verbal or visuospatial information and to retrieve information more fluently.

The improvements in memory performance, working memory and verbal fluency performance show the transfer ef- 
fect of improvement to cognitive domains other than memory. First, we expected that memory training would help increase memory performance and overcome the limitations of multistrategic training in the transfer to different cognitive domains or everyday cognitive tasks., ${ }^{2,4}$ This transfer effect may be related to improvements in higher cognitive functions and self-confidence in the memory process. Recent studies have shown the impact of the metamemory process on the facilitation of the memory process, enhancement of memory performance and transfer to new domains, suggesting that multistrategic training with a metamemory approach may facilitate encoding and retrieving through the alternative metamemory process including metaknowledge, metamonitoring and metajudgment. ${ }^{13,14,25}$ Second, we confirmed a positive relationship between objective changes in memory performance and the subjective perception of memory changes. ${ }^{15,26,27}$ Participant confidence in memory ability may be attributed to meta-monitoring of their own memory. ${ }^{26}$ Previous studies that were attempted to determine the enhancement of subjective feelings by multistrategic training have shown similar results as ours. ${ }^{5,6,26}$ Our study suggests that combining memory training with a holistic metamemory approach may improve higher strategic functions and self-efficacy as well as memory function in everyday life and social activities.

Another objective of our study was to determine whether depressive symptoms play crucial role in the outcomes of memory training. Depression is frequently associated with SMC and cognitive impairment. Based on the results of previous studies that changes in depressive symptoms affect cognitive function, our study excluded subjects with depressive symptoms and measured changes in cognitive performance., ${ }^{4,28}$ Previous longitudinal studies have indicated that SMC are more often associated with depressive symptoms. ${ }^{29,30}$ Our study provides a positive result that improvements in memory and other cognitive functions may be attributed to the effect of memory training rather than a decrease in depressive symptoms.

This study has some limitations. First, we did not examine how long the effect can be maintained. Second, our study has a limitation stemming from its small sample size. Further studies with a large sample size are needed to confirm that memory training with a metamemory approach affects daily life activities and how long this effect is maintained. Analytical methods in our study were adequately powered but we did not adjust for multiple comparisons, which might make some of type I errors. To our knowledge, this is the first study that showed the effect of specialized memory training with the metamemory concept for a normal elderly Korean population. Our method for memory training will help the normal elderly improve their quality of life.

\section{Acknowledgments}

This research was supported by Basic Science Research Program through the National Research Foundation of Korea (NRF) funded by the Ministry of Education, Science and Technology (Grant No. 20100025695).

\section{REFERENCES}

1. Park MH, Min JY, Min HY, Lee HJ, Lee DH, Song MS. Subjective memory complaints and clinical characteristics in elderly Koreans: a questionnaire survey. Int J Nurs Stud 2007;44:1400-1405.

2. Ball K, Berch DB, Helmers KF, Jobe JB, Leveck MD, Marsiske M, et al. Effects of cognitive training interventions with older adults: a randomized controlled trial. JAMA 2002;288:2271-2281.

3. Craik FI, Winocur G, Palmer H, Binns MA, Edwards M, Bridges K, et al. Cognitive rehabilitation in the elderly: effects on memory. J Int Neuropsychol Soc 2007;13:132-142.

4. Lustig C, Shah P, Seidler R, Reuter-Lorenz PA. Aging, training, and the brain: a review and future directions. Neuropsychol Rev 2009;19:504-522.

5. Stuss DT, Robertson IH, Craik FI, Levine B, Alexander MP, Black S, et al. Cognitive rehabilitation in the elderly: a randomized trial to evaluate a new protocol. J Int Neuropsychol Soc 2007;13:120-131.

6. Winocur G, Craik FI, Levine B, Robertson IH, Binns MA, Alexander $\mathrm{M}$, et al. Cognitive rehabilitation in the elderly: overview and future directions. J Int Neuropsychol Soc 2007;13:166-171.

7. Rebok GW, Carlson MC, Langbaum JB. Training and maintaining memory abilities in healthy older adults: traditional and novel approaches. J Gerontol B Psychol Sci Soc Sci 2007;62 Spec No 1:53-61.

8. Jonker C, Geerlings MI, Schmand B. Are memory complaints predictive for dementia? A review of clinical and population-based studies. Int J Geriatr Psychiatry 2000;15:983-991.

9. Jorm AF, Christensen H, Korten AE, Jacomb PA, Henderson AS. Memory complaints as a precursor of memory impairment in older people: a longitudinal analysis over 7-8 years. Psychol Med 2001;31: 441-449.

10. Flavell JH. Metacognition and cognitive monitoring: a new area of cognitive developmental inquiry. Am Psychol 1979;34:906-911.

11. Hertzog C, Dixon RA, Hultsch DF. Relationships between metamemory, memory predictions, and memory task performance in adults. Psychol Aging 1990;5:215-227.

12. Cavanaugh JC. Memory Self-Efficacy As a Key to Understanding Change. In: Blanchard-Fields F, Hess TM, Editors. Perspectives on Cognitive Change in Adulthood and Aging. New York: McGraw-Hill, 1996, p.488-507.

13. Koriat A, Bjork RA. Mending metacognitive illusions: a comparison of mnemonic-based and theory-based procedures. J Exp Psychol Learn Mem Cogn 2006;32:1133-1145.

14. Koriat A. Easy comes, easy goes? The link between learning and remembering and its exploitation in metacognition. Mem Cognit 2008; 36:416-428.

15. McDougall GJ, Kang J. Memory Self-Efficacy and Memory Performance in Older Males. Int J Mens Health. 2003;2:131-147.

16. Lee JH, Lee KU, Lee DY, Kim KW, Jhoo JH, Kim JH, et al. Development of the Korean version of the Consortium to Establish a Registry for Alzheimer's Disease Assessment Packet (CERAD-K): clinical and neuropsychological assessment batteries. J Gerontol B Psychol Sci Soc Sci 2002;57:47-53.

17. Morris JC, Heyman A, Mohs RC, Hughes JP, van Belle G, Fillenbaum $\mathrm{G}$, et al. The Consortium to Establish a Registry for Alzheimer's Disease (CERAD). Part I. Clinical and neuropsychological assessment of Alzheimer's disease. Neurology 1989;39:1159-1165.

18. Bae JN, Cho MJ. Development of the Korean version of the Geriatric Depression Scale and its short form among elderly psychiatric patients. J Psychosom Res 2004;57:297-305.

19. Ku HM, Kim JH, Kwon EJ, Kim SH, Lee HS, Ko HJ, et al. A study on 
the reliability and validity of Seoul-Instrumental Activities of Daily Living (S-IADL). J Korean Neuropsychiatr Assoc 2004;43:189-199.

20. Amemrican Psychiatric Association. Diagnostic and Statistical Manual of Mental Disorders, Fourth Edition. Washington DC: American Psychiatric Press; 1994.

21. Chey JY, Lee JE, Kim MJ, Kim HY. Development and standarization of the Elderly Verbal Learning Test (EVLT). Korean J Psychol Gen 2006;25: 141-173.

22. Benton AL, Hamsher KD. Multilingual Aphasia Examination. lowa city: AJA Associates; 1989.

23. Mitrushina MN, Boone KB, D'Elia LF. Handbook of Normative Data for Neuropsyhological Assessment. New York: Oxford University Press; 1999.

24. Youn JC, Kim KW, Lee DY, Jhoo JH, Lee SB, Park JH, et al. Development of the Subjective Memory Complaints Questionnaire. Dement Geriatr Cogn Disord 2009;27:310-317.

25. Schwarz N. Meta-cognitive experiences in consumer judgment and decision making. J Consum Psychol 2004;14:332-348.
26. Valentijn SA, Hill RD, Van Hooren SA, Bosma H, Van Boxtel MP, Jolles J, et al. Memory self-efficacy predicts memory performance: results from a 6-year follow-up study. Psychol Aging 2006;21:165-172.

27. Lane CJ, Zelinski EM. Longitudinal hierarchical linear models of the memory functioning questionnaire. Psychol Aging 2003;18:38-53.

28. Valentijn SA, van Hooren SA, Bosma H, Touw DM, Jolles J, van Boxtel MP, et al. The effect of two types of memory training on subjective and objective memory performance in healthy individuals aged 55 years and older: a randomized controlled trial. Patient Educ Couns 2005;57: 106-114.

29. Geerlings MI, Schoevers RA, Beekman AT, Jonker C, Deeg DJ, Schmand B, et al. Depression and risk of cognitive decline and Alzheimer's disease. Results of two prospective community-based studies in the Netherlands. Br J Psychiatry 2000;176:568-575.

30. Wilson RS, Mendes De Leon CF, Bennett DA, Bienias JL, Evans DA. Depressive symptoms and cognitive decline in a community population of older persons. J Neurol Neurosurg Psychiatry 2004;75:126-129. 